\title{
The real graphene oxide revealed: stripping the oxidative debris from the graphene-like sheets
}

Neil R. Wilson ${ }^{1}$, Priyanka A. Pandey ${ }^{1}$, Joseph J. Lea ${ }^{2}$, Matthew Bates ${ }^{1}$, Ian A. Kinloch ${ }^{3}$, Robert J. Young ${ }^{3}$, and Jonathan P. Rourke

${ }^{1}$ Department of Physics, University of Warwick, Coventry, CV4 7AL, UK Neil.Wilson@warwick.ac.uk

${ }^{2}$ Department of Chemistry, University of Warwick, Coventry, CV4 7AL, UK

${ }^{3}$ School of Materials, The University of Manchester, Grosvenor Street, Manchester, M1 7HS, UK

\section{Neil.Wilson@warwick.ac.uk}

Graphene oxide (GO) provides a potential route to large quantities of graphene, is cheap to make in bulk and easy to process. It is also a starting point for further functionalisation to create chemically modified graphenes (CMGs) e.g. for use in composites, light-harvesting, or as sensors. Understanding the chemical and physical structure of GO is a necessary step in its controllable functionalisation for CMGs and potential complete reduction back to graphene.

We will provide compelling evidence that GO, as produced by the Hummers' method, is composed of functionalized graphene sheets decorated by strongly bound oxidative debris (also referred to as fulvic acids), which acts as a surfactant to stabilize aqueous GO suspensions. We will also show that the physical and chemical properties of the as-produced GO are strongly influenced by this oxidative debris (OD). ${ }^{1}$ This OD-functionalised graphene complex appears to be indefinitely stable in water, but the removal of the OD can simply be effected with a base wash, whereupon the more highly functionalized debris dissolves fully into water, leaving a suspension of functionalized sheets. Careful independent weighing of the graphene oxide after washing, and the removed oxidative debris, shows that roughly a third of the mass of our as-produced graphene oxide can be ascribed to OD. The remaining graphenelike sheets are oxidized, but at a much lower level than current models for GO suggest. The importance of the OD is demonstrated by the change in properties of aGO after its removal: unlike as-produced GO, the resultant base-washed graphene oxide is not easily suspended in water and is conducting.

Our results suggest that models for the structure of graphene oxide need revisiting. The oxidative debris non-covalently attached to as-produced GO has important implications for the synthesis and application of CMGs, particularly where direct covalent functionalisation of the graphene lattice is required.

\section{References}

[1].Rourke, J.P. et al., Angew. Chem. Int. Ed., 50, 3173-3177 (2011)

Figure

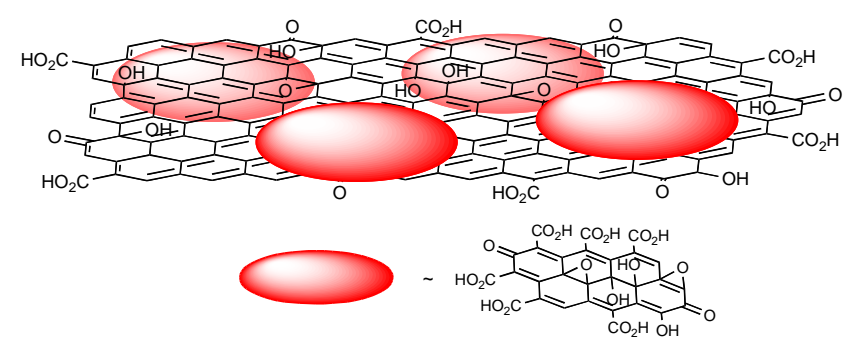

Figure. A diagrammatic representation of aGO; large oxidatively functionalized graphene-like sheets with surface bound debris. 